\title{
Publisher Correction: Social-ecological outcomes of agricultural intensification
}

Laura Vang Rasmussen, Brendan Coolsaet, Adrian Martin, Ole Mertz, Unai Pascual (D), Esteve Corbera (D), Neil Dawson, Janet A. Fisher, Phil Franks and Casey M. Ryan (1D

Correction to: Nature Sustainability https://doi.org/10.1038/s41893-018-0070-8, published online 14 June 2018.

In the version of this Review originally published, the top heading in the first column of Fig. 2 was mistakenly written 'Food poisoning'; it should have read 'Food provisioning. This has now been corrected. 